C2008 IEEE. Personal use of this material is permitted. However, permission to reprint/republish this material for advertising or promotional purposes or for creating new collective works for resale or redistribution to servers or lists, or to reuse any copyrighted component of this work in other works must be obtained from the IEEE. 


\title{
Optimal Design of All-Pass Variable Fractional-Delay Digital Filters
}

\author{
Wei Rong Lee, Lou Caccetta, and Volker Rehbock
}

\begin{abstract}
This paper presents a computational method for the optimal design of all-pass variable fractional-delay (VFD) filters aiming to minimize the squared error of the fractional group delay subject to a low level of squared error in the phase response. The constrained optimization problem thus formulated is converted to an unconstrained least-squares (LS) optimization problem which is highly nonlinear. However, it can be approximated by a linear LS optimization problem which in turn simply requires the solution of a linear system. The proposed method can efficiently minimize the total error energy of the fractional group delay while maintaining constraints on the level of the error energy of the phase response. To make the error distribution as flat as possible, a weighted LS (WLS) design method is also developed. An error weighting function is obtained according to the solution of the previous constrained LS design. The maximum peak error is then further reduced by an iterative updating of the error weighting function. Numerical examples are included in order to compare the performance of the filters designed using the proposed methods with those designed by several existing methods.
\end{abstract}

Index Terms-All-pass filter, constrained least-squares (LS) design, variable fractional-delay (VFD) filters, weighted LS (WLS) design.

\section{INTRODUCTION}

$\mathbf{V}$ ARIABLE fractional-delay (VFD) filters are filters with a variable fractional group delay. During the last decade, the design and implementation of such filters have received considerable attention due to their many applications in signal processing such as in communication, array processing, speech processing, and music technology [1]-[9]. A survey of fractional-delay filter design is presented in the tutorial paper [10]. VFD filters can be designed either using finite-impulse response (FIR) filters or infinite-impulse response (IIR) all-pass filters. So far, many methods have been developed for designing and implementing FIR VFD filters because of their relative simplicity [6], [7], [11]-[15]. In contrast, all-pass VFD filters are much more difficult to design. Because all-pass VFD filters are IIR filters, stability issue must be taken into account and the design of such filters usually involves nonlinear optimization. However, all-pass VFD filters have some advantages. Firstly, they have unity magnitude at all frequencies which is desirable in some applications. Secondly, the complexity of VFD filters, that is, the number of multipliers and delays required to implement the overall filters, is significant smaller for all-pass VFD filters when compared to FIR VFD filters [16]. Recently, several methods have been developed to design all-pass VFD filters [17]-[21]. In [17] and [18], Makundi et al. have introduced a so-called gathering structure, and a closed form method

\footnotetext{
Manuscript received March 7, 2007; revised September 13, 2007. This paper was recommended by Associate Editor P. Regalia.

The authors are with the Western Australian Centre of Excellence in Industrial Optimisation, Department of Mathematics andStatistics, Curtin University of Technology, Perth 6845, Australia (e-mail: liwr@maths.curtin.edu.au).

Digital Object Identifier 10.1109/TCSI.2008.916686
}

has been developed. In this structure, the filter coefficients are polynomials of the fractional-delay parameter. The method provides an analytic solution and is easy to use. However, the solution obtained in this method is not optimal. To increase the design accuracy, Tseng in [19] has extended the method developed in [22] and [23] for all-pass fixed fractional-delay filters to the all-pass VFD filter case. The method can achieve higher accuracy when compared with the previous methods. However, the algorithm proposed in this method does not converge in some cases. To further improve the method, Deng in [20] has developed a noniterative weighted least-squares (WLS) design method. In this method, a weighted squared error of variable frequency response with fixed denominator is used as the cost function to be minimized. The method can achieve even higher accuracy solutions than the method proposed in [19]. However, there are no clear rules to determine an appropriate weighting function.

In this paper, we have developed a new method for the all-pass VFD filter design. Firstly, we reformulate the design problem. The cost function chosen to be minimized is the squared error of the fractional group delay, since the group-delay error should be the major consideration when designing a nonlinear phase digital filter. In contrast, the cost function in [19] is the squared error of the phase response and the cost function in [20] is the weighted squared error of the modified frequency response (with fixed denominator). Our method directly minimizes the squared error of the fractional group delay and at the same time keeps the squared error of the phase response at a low level. The constrained LS optimization problem thus formulated is then converted to an ULS optimization problem. The ULS optimization problem is highly nonlinear. Fortunately, it can be effectively approximated by a linear LS optimization problem which is then solved via the solution of a linear system. To make the error distribution as flat as possible, many WLS methods have been proposed; see [19], [20], [27], [29], and [30]. In this paper, a WLS design is also developed. A weighting function is initially obtained according to the solution of the previous constrained LS design. The maximum peak error is then further reduced through an iterative update of the weighting function.

The paper is organized as follows. The constrained LS design problem is formulated in Section II. The solution method for the problem is developed in Section III. The WLS design method is then developed to further reduce the peak error in Section IV. In Section V an all-pass VFD filter is designed using the proposed methods. Section VI concludes the paper.

\section{CONSTRAINED LS DESIGN}

Let the desired frequency response of a VFD filter be

$$
H_{d}(w, p)=e^{-j(I+p) w} .
$$


Here, $D(w, p)=-(I+p) w$ is the desired phase response, $I$ is a fixed integer group delay, $p$ is a VFD in the range

$$
p \in[-0.5,0.5]
$$

and $w$ is the angular frequency in the range

$$
w \in[-\alpha \pi, \alpha \pi]
$$

where $0<\alpha<1$.

The VFD filter chosen to approximate $H_{d}(w, p)$ is an $N$ th-order all-pass filter with the transfer function $H(z, p)$ given by

$$
\begin{aligned}
H(z, p) & =\frac{a_{N}(p)+\cdots+a_{2}(p) z^{-N+1}+z^{-N}}{1+a_{1}(p) z^{-1}+a_{2}(p) z^{-2}+\cdots+a_{N}(p) z^{-N}} \\
& =z^{-N} \frac{A\left(z^{-1}, p\right)}{A(z, p)}
\end{aligned}
$$

where the denominator $A(z, p)$ is defined by

$$
A(z, p)=\sum_{n=0}^{N} a_{n}(p) z^{-n} .
$$

Here, the first coefficient $a_{0}(p)$ of $A(z, p)$ is fixed to be one and the remaining ones $\left\{a_{n}(p), n=1, \cdots, N\right\}$ are variable. Because of the mirror symmetric property of all-pass filters, the phase response $\theta(w, p)$ and the group delay $\tau(w, p)$ of this allpass VFD filter are

$$
\begin{aligned}
\theta(w, p) & =-N w-2 \arg [A(w, p)] \\
\tau(w, p) & =-\frac{d}{d w} \theta(w, p) \\
& =N+2 \frac{d}{d w} \arg [A(w, p)]
\end{aligned}
$$

respectively, where $A(w, p)$ is the frequency response of $A(z, p)$.

The variable coefficient $a_{n}(p)$ is assumed to be a polynomial function of the parameter $p$

$$
a_{n}(p)=\sum_{m=1}^{M} b_{n, m} p^{m}, \quad \text { for } \quad n=1, \cdots, N .
$$

Here, $M$ is the degree of the polynomials. The coefficient constraint proposed in [20], i.e., $b_{n, 0}=0$, for $n=1, \cdots, N$ is also imposed here to reduce the filter complexity. Our objective is to find the coefficients $\left\{b_{n, m}, n=1, \cdots, N, m=1, \cdots, M\right\}$ such that the error energy of the fractional group delay is minimized while the error energy of the phase response is kept low.

Define the error functions in the phase response and the fractional group delay as

$$
\begin{aligned}
\theta_{e}(w, p) & =\theta(w, p)-D(w, p) \\
\tau_{e}(w, p) & =-\frac{d}{d w} \theta_{e}(w, p) \\
& =-\frac{d}{d w}(\theta(w, p)-D(w, p))
\end{aligned}
$$

respectively. The design problem can then be formulated as: find the filter coefficients $\left\{b_{n, m}, n=1, \cdots, N, m=1, \cdots, M\right\}$ to minimize

$$
\int_{0}^{\alpha \pi} \int_{-0.5}^{0.5}\left(\tau_{e}(w, p)\right)^{2} d p d w
$$

such that

$$
\int_{0}^{\alpha \pi} \int_{-0.5}^{0.5}\left(\theta_{e}(w, p)\right)^{2} d p d w \leq \delta
$$

where $\delta$ is a small positive number. Here, the lower limits of integration with respect to the frequency $w$ for the both integrals in (4) and (6) are taken to be zero because of the symmetric property of both integrands. Let this constrained LS (CLS) optimization problem be referred to as Problem CLS . Because the fractional group-delay error should be the major consideration, the cost function (4) is to be minimized, and, at the same time, the error energy (5) of the phase response is kept at a low level.

\section{SOLUTION METHOD}

To solve Problem CLS, we append constraint (5) to the cost function (4) as a penalty, so thus we now minimize

$$
\int_{0}^{\alpha \pi} \int_{-0.5}^{0.5}\left\{\left(\tau_{e}(w, p)\right)^{2}+\zeta\left(\theta_{e}(w, p)\right)^{2}\right\} d p d w
$$

where the positive multiplier $\zeta$ controls the contribution of the constraint penalty term. $\zeta$ needs to be chosen sufficiently large so that the solution obtained from minimizing (6) satisfies the constraint (5). We start by picking a value for $\zeta$ and minimizing (6). If the resulting error energy of the phase response is greater than $\delta$, we can increase $\zeta$ and minimize (6) again. If it is much lower than $\delta$, we can reduce $\zeta$ and minimize (6) again. This process can be repeated several times until the constraint (5) is just satisfied. For each value of $\zeta$, Problem CLS is converted to the following ULS optimization problem: given a positive multiplier $\zeta$, find the filter coefficients $\left\{b_{n, m}, n=1, \cdots, N, m=\right.$ $1, \cdots, M\}$ to minimize (6). Let this ULS optimization problem be referred to as Problem ULS.

The frequency response $H(w, p)$ of $H(z, p)$ can be expressed as

$$
\begin{aligned}
H(w, p) & =e^{-j(I+p) w} e^{j(-N+I+p) w} \frac{\sum_{n=0}^{N} a_{n}(p) e^{j n w}}{\sum_{n=0}^{N} a_{n}(p) e^{-j n w}} \\
& =e^{-j(I+p) w} \frac{\sum_{n=0}^{N} a_{n}(p) e^{j\left(n w-\frac{N-I-p}{2} w\right)}}{\sum_{n=0}^{N} a_{n}(p) e^{-j\left(n w-\frac{N-I-p}{2} w\right)}} \\
& =e^{-j(I+p) w} \frac{\sum_{n=0}^{N} a_{n}(p) e^{j \Theta_{n}(w, p)}}{\sum_{n=0}^{N} a_{n}(p) e^{-j \Theta_{n}(w, p)}}
\end{aligned}
$$

where $\Theta_{n}(w, p)=n w-(N-I-p / 2) w$. Because of the symmetry property of this filter, the phase response $\theta(w, p)$ of 
$H(w, p)$ can be given as

$\theta(w, p)=-(I+p) w+2 \tan ^{-1} \frac{\sum_{n=0}^{N} a_{n}(p) \sin \left(\Theta_{n}(w, p)\right)}{\sum_{n=0}^{N} a_{n}(p) \cos \left(\Theta_{n}(w, p)\right)}$.

For an all-pass VFD filter to be stable, it is desirable that $\theta(w, p)$ has the following properties [24]: 1) $\theta(0, p)=0$ for $p \in[-0.5,0.5]$; 2) $\theta(\pi, p)=-N \pi$ for $p \in[-0.5,0.5]$; and 3) $\theta(w, p)$ decreases monotonically with $w$ for each $p \in[-0.5,0.5]$. To approximate these requirements well, we let $N=I$. If an all-pass VFD filter approximates the desired one accurately, the first and third requirements will be satisfied, and the absolute phase-response error of this filter at frequency $w=\pi$ for all $p \in[-0.5,0.5]$ will be less than $\pi$. As pointed out by Jing in [25], the designed all-pass VFD filter will be stable in this case. Since $N=I$, the above equation for $\Theta_{n}(w, p)$ simplifies to $\Theta_{n}(w, p)=n w+(p / 2) w$.

From (8), the phase-response error $\theta_{e}(w, p)$ in (2) can be specified as

or

$$
\theta_{e}(w, p)=2 \tan ^{-1} \frac{\sum_{n=0}^{N} a_{n}(p) \sin \left(\Theta_{n}(w)\right)}{\sum_{n=0}^{N} a_{n}(p) \cos \left(\Theta_{n}(w, p)\right)}
$$

$$
\theta_{e}(w, p)=2 \sin ^{-1} \frac{\sum_{n=0}^{N} a_{n}(p) \sin \left(\Theta_{n}(w, p)\right)}{\rho(w, p)}
$$

where $\rho(w, p)=\left\{\left(\sum_{n=0}^{N} a_{n}(p) \sin \left(\Theta_{n}(w, p)\right)\right)^{2}+\right.$ $\left.\left(\sum_{n=0}^{N} a_{n}(p) \cos \left(\Theta_{n}(w, p)\right)\right)^{2}\right\}^{1 / 2}$ is the magnitude of the frequency response $A(w, p)$. When the absolute value of $\theta_{e}(w, p)$ is very close to zero, the term $\theta_{e}(w, p)$ in (10) can be readily approximated by

$$
\theta_{e}(w, p)=2 \frac{\sum_{n=0}^{N} a_{n}(p) \sin \left(\Theta_{n}(w, p)\right)}{\rho(w, p)} .
$$

Since $\int_{0}^{\alpha \pi} \int_{-0.5}^{0.5}\left\{\zeta\left(\theta_{e}(w, p)\right)^{2}\right\} d p d w$ in the cost function (6) will be minimized as a penalty term, and since the absolute value of $\theta_{e}(w, p)$ will be very small for all points in $\{(w, p) \mid w \in$ $[0, \alpha \pi], p \in[-0.5,0.5]\}$, the above approximation is justified at the solution of this optimization problem.

Substituting (9) into (3), we have

$$
\begin{aligned}
\tau_{e}(w, p)= & -\frac{d}{d w} \theta_{e}(w, p) \\
= & \frac{-2 \operatorname{Re}(w, p)}{(\rho(w, p))^{2}} \sum_{n=0}^{N}\left(\left(n+\frac{p}{2}\right) a_{n}(p) \cos \left(\Theta_{n}(w, p)\right)\right. \\
& +\frac{-2 \operatorname{Im}(w, p)}{(\rho(w, p))^{2}} \\
& \times \sum_{n=0}^{N}\left(\left(n+\frac{p}{2}\right) a_{n}(p) \sin \left(\Theta_{n}(w, p)\right)\right.
\end{aligned}
$$

where

$$
\operatorname{Re}(w, p)=\sum_{n=0}^{N} a_{n}(p) \cos \left(\Theta_{n}(w, p)\right)
$$

and

$$
\operatorname{Im}(w, p)=\sum_{n=0}^{N} a_{n}(p) \sin \left(\Theta_{n}(w, p)\right)
$$

are the real and imaginary parts of the frequency response $A(w, p)$, respectively.

Since $\left|\theta_{e}(w, p)\right|$ is very small as mentioned above, the term $\left|\operatorname{Im}(w, p) /(\rho(w, p))^{2}\right|$ is also very small, and $\operatorname{Re}(w, p) /(\rho(w, p))^{2} \mid \approx \sqrt{\left((\operatorname{Re}(w, p))^{2}+(\operatorname{Im}(w, p))^{2}\right) /(\rho(w, p))^{4}}$ $=1 / \rho(w, p)$ is very large compared to the term $\left|\operatorname{Im}(w, p) /(\rho(w, p))^{2}\right|$ for all points in $\{(w, p) \mid w \in[0, \alpha \pi], p \in[-0.5,0.5]\}$. Consequently, the error of the group delay in (12) is dominated by the term $\left(-2 \operatorname{Re}(w, p) /(\rho(w, p))^{2}\right) \sum_{n=0}^{N}((n \quad+$ $(p / 2)) a_{n}(p) \cos \left(\Theta_{n}(w, p)\right)$. We can therefore approximate $\tau_{e}(w, p)$ in (12) with

$\tau_{e}(w, p)=\frac{-2 \operatorname{Re}(w, p)}{(\rho(w, p))^{2}} \sum_{n=0}^{N}\left(\left(n+\frac{p}{2}\right) a_{n}(p) \cos \left(\Theta_{n}(w, p)\right)\right.$.

Furthermore, we note that

$$
\left|\tau_{e}(w, p)\right| \approx 2 \frac{\sum_{n=0}^{N}\left(\left(n+\frac{p}{2}\right) a_{n}(p) \cos \left(\Theta_{n}(w, p)\right)\right.}{\rho(w, p)} .
$$

We then have the following simplified version of Problem ULS: given a value of $\zeta$, find the filter coefficients $\left\{b_{n, m}, n=\right.$ $1, \cdots, N, m=1, \cdots, M\}$ such that

$$
\begin{gathered}
\int_{0}^{\alpha \pi} \int_{-0.5}^{0.5}\left\{\frac{\left(\sum_{n=0}^{N}\left(n+\frac{p}{2}\right) a_{n}(p) \cos \left(\Theta_{n}(w, p)\right)\right)^{2}}{\rho^{2}(w, p)}\right. \\
\left.+\frac{\zeta\left(\sum_{n=0}^{N} a_{n}(p) \sin \left(\Theta_{n}(w, p)\right)\right)^{2}}{\rho^{2}(w, p)}\right\} d p d w
\end{gathered}
$$

is minimized. Here, we omit the multiplier 4, which has no influence on the optimization process.

Because of the presence of the denominator term $\rho^{2}(w, p)$ in (15), the above LS optimization problem is nonlinear. To overcome this difficulty, Tseng, in [19], has introduced a method based on the so-called Steiglitz-McBride iteration [26]. The method can achieve higher design accuracy than the maximally flat design in [18] even in the high-frequency band. However, the convergence properties of Steiglitz-McBride iteration have not been established yet, see [27] and [28]. As we can observe from Fig. 1, the magnitude $\rho(w, p)$ of the frequency response $A(w, p)$ in the range $\{(w, p) \mid w \in[0, \alpha \pi], p \in[-0.5,0.5]\}$, which is obtained from minimizing (15) with $\rho(w, p)$ fixed to be 1 , is always close to 1 , thus suggesting a stable filter. We therefore approximate $\rho(w, p)$ in (15) with the constant number 1 . This is equivalent to multiplying the integrand in (15) by $\rho^{2}(w, p)$, resulting in a WLS design with $\rho^{2}(w, p)$ as a weighting function. Problem ULS can then be simplified, resulting in a modified Problem ULS: given a specified $\zeta$, find the filter coefficients 


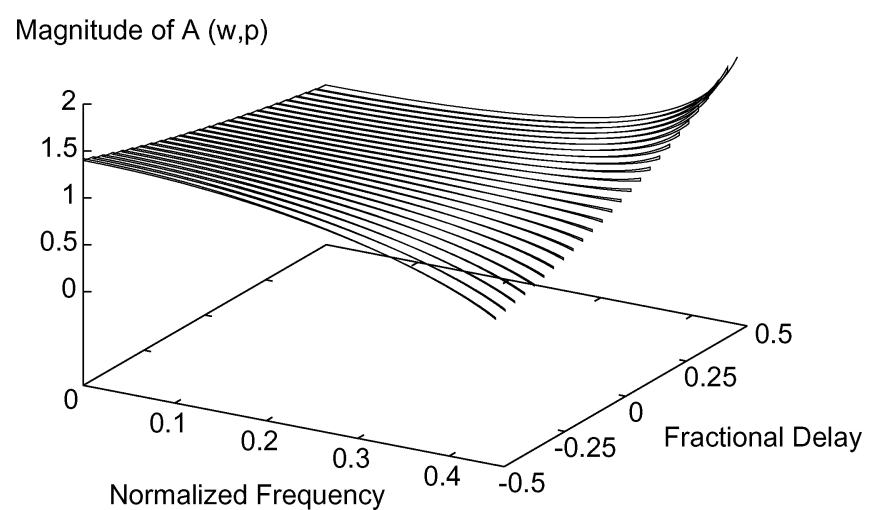

Fig. 1. Magnitude of the frequency response $A(w, p)$.

$\left\{b_{n, m}, n=1, \cdots, N, m=1, \cdots, M\right\}$ such that

$$
J_{1}=\int_{0}^{\alpha \pi} \int_{-0.5}^{0.5}\left\{E^{2}+\zeta F^{2}\right\} d p d w
$$

is minimized. Here

$$
\begin{aligned}
E & =\sum_{n=0}^{N}\left(n+\frac{p}{2}\right) a_{n}(p) \cos \left(\Theta_{n}(w, p)\right) \\
F & =\sum_{n=0}^{N} a_{n}(p) \sin \left(\Theta_{n}(w, p)\right) .
\end{aligned}
$$

Substituting (1) into (16) and (17), we have

$$
\begin{aligned}
& E=\boldsymbol{c}(w, p) B \boldsymbol{p}^{\top}+\left(\frac{p}{2} w\right) \cos \left(\frac{p}{2} w\right) \\
& F=\boldsymbol{s}(w, p) B \boldsymbol{p}^{\top}+\sin \left(\frac{p}{2} w\right)
\end{aligned}
$$

respectively, where $\boldsymbol{c}(w, p) \quad=\quad[(1+$ $\left.(p / 2)) \cos \left(\Theta_{1}(w, p)\right), \ldots,(N+(p / 2)) \cos \left(\Theta_{N}(w, p)\right)\right]$, $\boldsymbol{s}(w, p)=\left[\sin \left(\Theta_{1}(w, p)\right), \ldots, \sin \left(\Theta_{N}(w, p)\right)\right]$, $\boldsymbol{p}=\left[p, \ldots, p^{M}\right], \top$ stands for transposition and

$$
B=\left[\begin{array}{ccc}
b_{1,1} & \ldots & b_{1, M} \\
\vdots & \ddots & \vdots \\
b_{N, 1} & \ldots & b_{N, M}
\end{array}\right]
$$

It is easy to verify that $\boldsymbol{c}(w, p) B \boldsymbol{p}^{\top}$ and $\boldsymbol{s}(w, p) B \boldsymbol{p}^{\top}$ can be written as

$$
\begin{aligned}
\boldsymbol{c}(w, p) B \boldsymbol{p}^{\top} & =\overline{\boldsymbol{c}}(w, p) \boldsymbol{b}^{\top} \\
\boldsymbol{s}(w, p) B \boldsymbol{p}^{\top} & =\overline{\boldsymbol{s}}(w, p) \boldsymbol{b}^{\top}
\end{aligned}
$$

respectively, where

$$
\begin{aligned}
\overline{\boldsymbol{c}}(w, p) & =\left[\boldsymbol{c}(w, p), \boldsymbol{c}(w, p) p, \ldots, \boldsymbol{c}(w, p) p^{M}\right] \\
\overline{\boldsymbol{s}}(w, p) & =\left[\boldsymbol{s}(w, p), \boldsymbol{s}(w, p) p, \ldots, \boldsymbol{s}(w, p) p^{M}\right] \\
\boldsymbol{b} & =\left[b_{1,1} \ldots b_{N, 1} b_{1,2} \ldots b_{N, 2} \ldots b_{1, M} \ldots b_{N, M}\right] .
\end{aligned}
$$

Since $\overline{\boldsymbol{c}}(w, p)$ and $\overline{\boldsymbol{s}}(w, p)$ are both $N M \times 1$ vectors, we can rename their components as follows:

$$
\begin{aligned}
\overline{\boldsymbol{c}}(w, p) & =\left[\bar{c}_{1}(w, p), \ldots, \bar{c}_{N M}(w, p)\right] \\
\overline{\boldsymbol{s}}(w, p) & =\left[\bar{s}_{1}(w, p), \ldots, \bar{s}_{N M}(w, p)\right] .
\end{aligned}
$$

Hence, the cost function $J_{1}$ can be rewritten as

$$
\begin{gathered}
J_{1}=\int_{0}^{\alpha \pi} \int_{-0.5}^{0.5}\left\{\left[\overline{\boldsymbol{c}}(w, p) \boldsymbol{b}^{\top}+\left(\frac{p}{2} w\right) \cos \left(\frac{p}{2} w\right)\right]^{2}\right. \\
\left.+\zeta\left[\overline{\boldsymbol{s}}(w, p) \boldsymbol{b}^{\top}+\sin \left(\frac{p}{2} w\right)\right]^{2}\right\} d p d w \\
=\boldsymbol{b} G \boldsymbol{b}^{\top}+2 \boldsymbol{g} \boldsymbol{b}^{\top}+C
\end{gathered}
$$

where the $N M \times N M$ matrix $G=\left[G_{i, j}\right]$, the $N M \times 1$ vector $g=\left\{g_{1}, \cdots, g_{N M}\right\}^{\top}$ and the scalar $C$ are given by

$$
\begin{aligned}
& G_{i, j}= \int_{0}^{\alpha \pi} \int_{-0.5}^{0.5}\left\{\bar{c}_{i}(w, p) \bar{c}_{j}(w, p)\right. \\
& g_{i}=\int_{0}^{\alpha \pi} \int_{-0.5}^{0.5}\left\{\bar{c}_{i}(w, p)\left(\frac{p}{2} w\right) \cos \left(\frac{p}{2} w\right)\right. \\
&\left.+\zeta \bar{s}_{i}(w, p) \sin \left(\frac{p}{2} w\right)\right\} d p d w
\end{aligned}
$$

and

$$
C=\int_{0}^{\alpha \pi} \int_{-0.5}^{0.5}\left\{\left[\frac{p}{2} w \cos \left(\frac{p}{2} w\right)\right]^{2}+\zeta\left[\sin \left(\frac{p}{2} w\right)\right]^{2}\right\} d p d w
$$

respectively. Because $J_{1}$ is a quadratic function in $\boldsymbol{b}$, the optimal solution of the modified Problem ULS is unique and can be obtained by solving the following linear system:

$$
G \boldsymbol{b}^{\top}=-\boldsymbol{g} .
$$

The Matrix $G$ is symmetric and positive definite. Consequently, it is nonsingular and has a Cholesky factorization. The linear system (22) can therefore be readily solved.

\section{WLS DESIGN}

To make the error distribution as flat as possible, we define a new objective function

$$
\begin{aligned}
J_{2}=\int_{0}^{\alpha \pi} \int_{-0.5}^{0.5}\left\{W(w, p)\left[\overline{\boldsymbol{c}}(w, p) \boldsymbol{b}^{\top}+\left(\frac{p}{2} w\right) \cos \left(\frac{p}{2} w\right)\right]^{2}\right. \\
\left.+\zeta^{*}\left[\overline{\boldsymbol{s}}(w, p) \boldsymbol{b}^{\top}+\sin \left(\frac{p}{2} w\right)\right]^{2}\right\} d p d w
\end{aligned}
$$

where $W(w, p)$ is a weighting function and $\zeta^{*}$ is a specified value of $\zeta$. If $W(w, p)=1$ in (23), $J_{2}$ is reduced to $J_{1}$ with $\zeta=\zeta^{*}$. The corresponding WLS optimization problem can be 
formulated as: given a specified $\zeta^{*}$ and a weighting function $W(w, p)$, find the filter coefficients $\left\{b_{n, m}, n=1, \cdots, N, m=\right.$ $1, \cdots, M\}$ such that the cost function (23) is minimized. Let this problem be referred to as Problem WLS. Given a specified $\zeta^{*}$, our objective in this section is to find an appropriate weighting function $W(w, p)$ and solve the corresponding Problem WLS to further reduce the peak error of the fractional group delay obtained from the solution of the modified Problem ULS with $\zeta=\zeta^{*}$. For simplicity, we will use the name 'Problem ULS' instead of the name 'the modified Problem ULS' from now on.

We now introduce an adaptive weighing function, which is based on the optimal solution of Problem ULS. Let $\boldsymbol{b}^{*}$ be the optimal solution of Problem ULS, and $a_{n}^{*}(p)$ be the corresponding value of $a_{n}(p)$ obtained from (1). Then, we chose

$$
W(w, p)= \begin{cases}\frac{\sigma(w, p)}{\gamma}, & \text { if } \sigma(w, p) \geq \gamma \\ 1, & \text { otherwise }\end{cases}
$$

where

$$
\begin{gathered}
\sigma(w, p)=\mid \frac{2 \operatorname{Re}(w, p)\left\{\sum_{n=0}^{N}\left(\left(n+\frac{p}{2}\right) a_{n}^{*}(p) \cos \left(\Theta_{n}(w)\right)\right\}\right.}{(\rho(w, p))^{2}} \\
+\frac{2 \operatorname{Im}(w, p)\left\{\sum_{n=0}^{N}\left(\left(n+\frac{p}{2}\right) a_{n}^{*}(p) \sin \left(\Theta_{n}(w)\right)\right\}\right.}{(\rho(w, p))^{2}} \mid \cdot \quad 25
\end{gathered}
$$

$\sigma(w, p)$ is the absolute value of the fractional group-delay error at point $(w, p)$ resulting from the solution $\boldsymbol{b}^{*}$ of Problem ULS and $\gamma$ is a fixed small positive number. $W(w, p)$ has several properties. Firstly, it is equal to one at most points except those where the absolute value of the group-delay error $\sigma(w, p)$ is larger than $\gamma$. Secondly, large weighting values will result at the points where large peak errors exist, i.e., the weight is concentrated on the large peak ripples. Obviously, the value of $\gamma$ affects the reduction of the peak error at the solution of Problem WLS. A large value of $\gamma$ will result in a small reduction of the peak error, and a small value of $\gamma$ could result in a large reduction of the peak error if the choice of the value of $\gamma$ is appropriate. From our numerical experience, a value of $\gamma$ between $30 \%$ and $40 \%$ of the maximum peak error corresponding to the solution $\boldsymbol{b}^{*}$ of Problem ULS is appropriate.

The peak error of the fractional group delay for the solution of Problem WLS will be reduced when compared with that of the solution of Problem ULS. A further improvement can be achieved if the first solution of Problem WLS is substituted into (25) (replacing the $\boldsymbol{b}^{*}$ ) and the corresponding $W(w, p)$ from (24) is then used to modulate the previous weighting function to produce an updated weighting function. Problem WLS is then solved again with this updated weighting function, resulting a flatter peak error distribution. This second step can be repeated several times to make the peak error distribution as flat as possible. The whole procedure can be summarized by the following algorithm:

Data: Choose a positive multiplier $\zeta^{*}$ for Problem ULS, and the number of times, $k$, that Problem WLS is to be solved.

Step 1) Find the optimal solution, $\boldsymbol{b}^{*}$, of Problem ULS.

Choose a small positive number $\gamma$ for formula (25).
Calculate the weighting function $W_{1}(w, p)$ using $\boldsymbol{b}^{*}$ and the formulas (24) and (25).

Step 2) Solve the Problem WLS with weighting function $W(w, p)=W_{1}(w, p)$ in (23). Let $\boldsymbol{b}^{(1)}$ be the optimal solution. Set $i=1$.

Step 3) Let $i=i+1$. Calculate $W_{i}(w, p)$ using $\boldsymbol{b}^{(i-1)}$ in place of $\boldsymbol{b}^{*}$ in the formulas (24) and (25).

Step 4) Solve the Problem WLS with weighting function $W(w, p)=\prod_{l=1}^{i} W_{l}(w, p)$ in (23). Let $\boldsymbol{b}^{(i)}$ be the optimal solution. If $i<k$, go to Step 3 .

Step 5) $W(w, p)=\prod_{l=1}^{k} W_{l}(w, p)$ is the final weighting function and $\boldsymbol{b}^{(\bar{k})}$ is the final WLS solution.

Remark 1: In Step 4), the weighting function $W(w, p)$ is the product of $W_{l}(w, p), l=1, \ldots, i$, since $W_{i}(w, p)$ obtained in Step 3) is based on the optimal solution of the previous Problem WLS which employed the weighting function $W(w, p)=\prod_{l=1}^{i-1} W_{l}(w, p)$.

Remark 2: The weighting function starts with $W_{1}(w, p)$, and is constructed iteratively. The convergence could be claimed when the difference, $\left\|\boldsymbol{b}^{i+1}-\boldsymbol{b}^{i}\right\|$, of two consecutive WLS solutions is less than a prescribed tolerance $\epsilon$. For practical applications, we usually only need about $4-8$ iterations to make the error distribution sufficiently flat; see Table I.

We now calculate a discretized version of the above weighting functions. Let $\Omega=\{(w, p) \mid, w \in[0, \alpha \pi], p \in[-0.5,0.5]\}$, be a dense set of points uniformly distributed in the region $[0, \alpha \pi] \times$ $[-0.5,0.5]$. If the total number of points in the set $\Omega$ is $L$, we can write the set as $\Omega=\left\{\left(w_{i}, p_{i}\right) \mid i=1, \cdots, L\right\}$. For a given $\zeta^{*}$, the optimal solution, $\boldsymbol{b}^{*}$, of the corresponding Problem ULS can be obtained immediately as stated in the previous section. The value of $W_{1}\left(w_{i}, p_{i}\right)$ at each $\left(w_{i}, p_{i}\right)$ for $i=1, \cdots, L$ can then be obtained from $\boldsymbol{b}^{*}$ and the formulae (24) and (25). $W_{l}(w, p)$ for $l=2, \cdots, k$ can be obtained in the same manner after the corresponding Problem WLS has been solved.

To solve Problem WLS with a given $\zeta^{*}$ and a weighting function $\left\{W\left(w_{i}, p_{i}\right) \mid i=1, \cdots, L\right\}$, we form the following overdetermined linear system:

$$
\Psi \tilde{G} \boldsymbol{b}^{\top}=-\Psi \tilde{g}
$$

where

$$
\Psi=\left[\begin{array}{cc}
\Psi_{1} & 0 \\
0 & \Psi_{2}
\end{array}\right] \text { and } \quad \tilde{G}=\left[\begin{array}{l}
\tilde{G}_{1} \\
\tilde{G}_{2}
\end{array}\right] \tilde{g}=\left[\begin{array}{l}
\tilde{g}_{1} \\
\tilde{g}_{2}
\end{array}\right] .
$$

Here, $\Psi_{1}=\operatorname{diag}\left\{W\left(w_{1}, p_{1}\right) \cdots W\left(w_{L}, p_{L}\right)\right\}, \Psi_{2}=$ $\operatorname{diag}\left\{\zeta^{*} \cdots \zeta^{*}\right\}$ are two $L \times L$ diagonal matrices

$$
\begin{array}{r}
\tilde{G}_{1}=\left[\begin{array}{ccc}
\bar{c}_{1}\left(w_{1}, p_{1}\right) & \cdots & \bar{c}_{N M}\left(w_{1}, p_{1}\right) \\
\vdots & \ddots & \vdots \\
\bar{c}_{1}\left(w_{L}, p_{L}\right) & \cdots & \bar{c}_{N M}\left(w_{L}, p_{L}\right)
\end{array}\right] \\
\tilde{G}_{2}=\left[\begin{array}{ccc}
\bar{s}_{1}\left(w_{1}, p_{1}\right) & \cdots & \bar{s}_{N M}\left(w_{1}, p_{1}\right) \\
\vdots & \ddots & \vdots \\
\bar{s}_{1}\left(w_{L}, p_{L}\right) & \cdots & \bar{s}_{N M}\left(w_{L}, p_{L}\right)
\end{array}\right]
\end{array}
$$

are two $N M \times L$ matrices, and $\tilde{g}_{1}=$ $\left[\left(\left(p_{1} / 2\right) w_{1}\right) \cos \left(\left(p_{1} / 2\right) w_{1}\right), \cdots,\left(\left(p_{L} / 2\right) w_{L}\right) \cos \left(\left(p_{L} / 2\right) w_{L}\right)\right]^{\top}$, 
TABLE I

The Design ERrors of Various Designs Using Various Methods

\begin{tabular}{l|l|l|l|l|r}
\hline \hline method & $\epsilon_{\tau 2}(\%)$ & $\epsilon_{\tau M a x}$ & $\epsilon_{\theta 2}(\%)$ & $\epsilon_{\theta M a x}$ & coefficients \\
\hline \hline $\begin{array}{l}\text { Proposed LS Design } \\
\text { with } \delta=0.0022 \text { for the constraint (5) }\end{array}$ & 0.0992 & 0.005276 & 0.002199 & 0.0000718 & 175 \\
\hline $\begin{array}{l}\text { Non-iterative } \\
\text { LS Design in [20] }\end{array}$ & 0.126 & 0.0158 & 0.0012 & 0.000179 & 175 \\
\hline $\begin{array}{l}\text { Iterative LS } \\
\text { design in [19] }\end{array}$ & 0.2693 & 0.0369 & 0.0024 & 0.000553 & 210 \\
\hline $\begin{array}{l}\text { Proposed WLS Design with } \\
\text { Problem (WLS) solved 2 times }\end{array}$ & 0.116 & 0.003407 & 0.00333 & 0.0001063 & 175 \\
$\begin{array}{l}\text { Proposed WLS Design with } \\
\text { Problem (WLS) solved 4 times }\end{array}$ & 0.142 & 0.003084 & 0.00324 & 0.0000956 & 175 \\
\hline $\begin{array}{l}\text { Proposed WLS Design with } \\
\text { Problem (WLS) solved 8 times }\end{array}$ & 0.1512 & 0.002889 & 0.00309 & 0.0000949 & 175 \\
\hline $\begin{array}{l}\text { Proposed WLS Design with } \\
\text { Problem (WLS) solved 16 times }\end{array}$ & 0.155 & 0.002836 & 0.00307 & 0.0000838 & 175 \\
\hline $\begin{array}{l}\text { Non-iterative WLS } \\
\text { Design in [20] }\end{array}$ & 0.0994 & 0.0067 & 0.0014 & 0.000048 & 175 \\
\hline $\begin{array}{l}\text { Iterative WLS } \\
\text { design in [19] }\end{array}$ & 0.2294 & 0.0291 & 0.0021 & 0.000422 & 210 \\
\hline
\end{tabular}

$\tilde{g}_{2}=\left[\sin \left(\left(p_{1} / 2\right) w_{1}\right), \cdots, \sin \left(\left(p_{L} / 2\right) w_{L}\right)\right]^{\top}$ are two $L \times 1$ vectors. The solution of Problem WLS can be obtained by solving the following linear system:

$$
\left(\tilde{G}^{\top} \Psi \tilde{G}\right) \boldsymbol{b}^{\top}=-\left(\tilde{G}^{\top} \Psi \tilde{g}\right) .
$$

Obviously, the matrix $\left(\tilde{G}^{\top} \Psi \tilde{G}\right)$ is symmetric and positive definite. The linear system (27) can therefore be readily solved.

Remark: Clearly, we could use another similar weighting function on the constrained penalty term to reduce the peak error of the phase response.

\section{DEsign EXAMPLE}

The design methods described in the previous sections are illustrated by the following 35th-order IIR all-pass VFD filter which was considered for testing the existing iterative and noniterative WLS methods in [19] and [20], respectively.

Example: The design specification is

$$
D(w, p)=-(35+p) w
$$

where

$$
p \in[-0.5,0.5] \quad w \in[-\alpha \pi, \alpha \pi] \quad \text { and } \quad \alpha=0.9 .
$$

The filter order and polynomial degree are

$$
\begin{aligned}
N & =35 \\
M & =5
\end{aligned}
$$

respectively, which are the same as those considered in [19] and [20].

The filter is designed using the proposed CLS design. The value $\delta$ for the constraint (5) is chosen to be 0.0022 . To solve this problem, the problem is converted to a sequence of Problems ULS. Initially, the penalty weight $\zeta_{1}$ is chosen to be 1 , and the corresponding Problem ULS is solved. The resulting error energy of the phase response is 0.00233 , which is larger than the allowed value 0.0022 . We then change the penalty value and solve the corresponding Problem ULS several times until the constraint (5) is just satisfied. The sequence of the penalty values used is $\left(\zeta_{1}=1, \zeta_{2}=10, \zeta_{3}=5, \zeta_{4}=3, \zeta_{5}=3.5\right.$ and $\zeta_{6}=3.3$ ). The corresponding sequence of the error energies of the phase response is $(0.0033,0.001996,0.002129,0.002213$, 0.002190 and 0.002199$)$. The final error energy of the phase response is 0.002199 which is just under the allowed value of 0.0022 .

The filter is also designed using the proposed WLS design. Problem ULS with $\zeta^{*}=10$ is initially solved. To create the weighting function (24) and (25) for Problem WLS, $\gamma$ is chosen to be 0.002 , because the maximum peak error of the fractionaldelay of the Problem ULS is 0.00528 . The number of times, $k$, that Problem WLS is to be solved are 2, 4, 8, and 16, respectively. To calculate the weighting functions and the solutions of Problem WLS, 201 sample points were used in the frequency range $[0, \alpha \pi]$ and 51 sample points were used in the parameter range $\{p \mid-0.5 \leq p \leq 0.5\}$ to discretize frequency value and parameter value, respectively.

To evaluate the accuracy of the filters designed, the normalized root mean square (RMS) fractional-delay error $\epsilon_{\tau 2}$ and maximum fractional-delay error $\epsilon_{\tau M a x}$ defined by

$$
\begin{aligned}
\epsilon_{\tau 2} & =\left[\frac{\int_{0}^{\alpha \pi} \int_{-0.5}^{0.5}\left(\tau_{e}(w, p)\right)^{2} d w d p}{\int_{0}^{\alpha \pi} \int_{-0.5}^{0.5}(p)^{2} d w d p}\right]^{\frac{1}{2}} 100(\%) \\
\epsilon_{\tau \text { Max }} & =\operatorname{Max}\left\{\left|\tau_{e}(w, p)\right| \mid w \in[0, \alpha \pi], p \in[-0.5,0.5]\right\}
\end{aligned}
$$

and the normalized RMS phase-response error $\epsilon_{\theta 2}$ and maximum phase-response error $\epsilon_{\theta M a x}$ defined by

$$
\begin{aligned}
\epsilon_{\theta 2} & =\left[\frac{\int_{0}^{\alpha \pi} \int_{-0.5}^{0.5}\left(\theta_{e}(w, p)\right)^{2} d w d p}{\int_{0}^{\alpha \pi} \int_{-0.5}^{0.5}(p w)^{2} d w d p}\right]^{\frac{1}{2}} 100(\%) \\
\epsilon_{\theta \text { Max }} & =\operatorname{Max}\left\{\left|\theta_{e}(w, p)\right| \mid w \in[0, \alpha \pi], p \in[-0.5,0.5]\right\}
\end{aligned}
$$
are used. In (28) and (29), $\tau_{e}(w, p)$ is defined by (3) and in (30) and (31), $\theta_{e}(w, p)$ is defined by (2). Table I lists the design errors of the proposed methods. For comparison, the design errors of the methods in [19] and [20] are also listed in the table. It can be 
TABLE II

COEFFICIENTS, $b_{n, m}$, OF THE DENOMINATOR $A(z, p)$ OF THE PROPOSED LS DESIGN

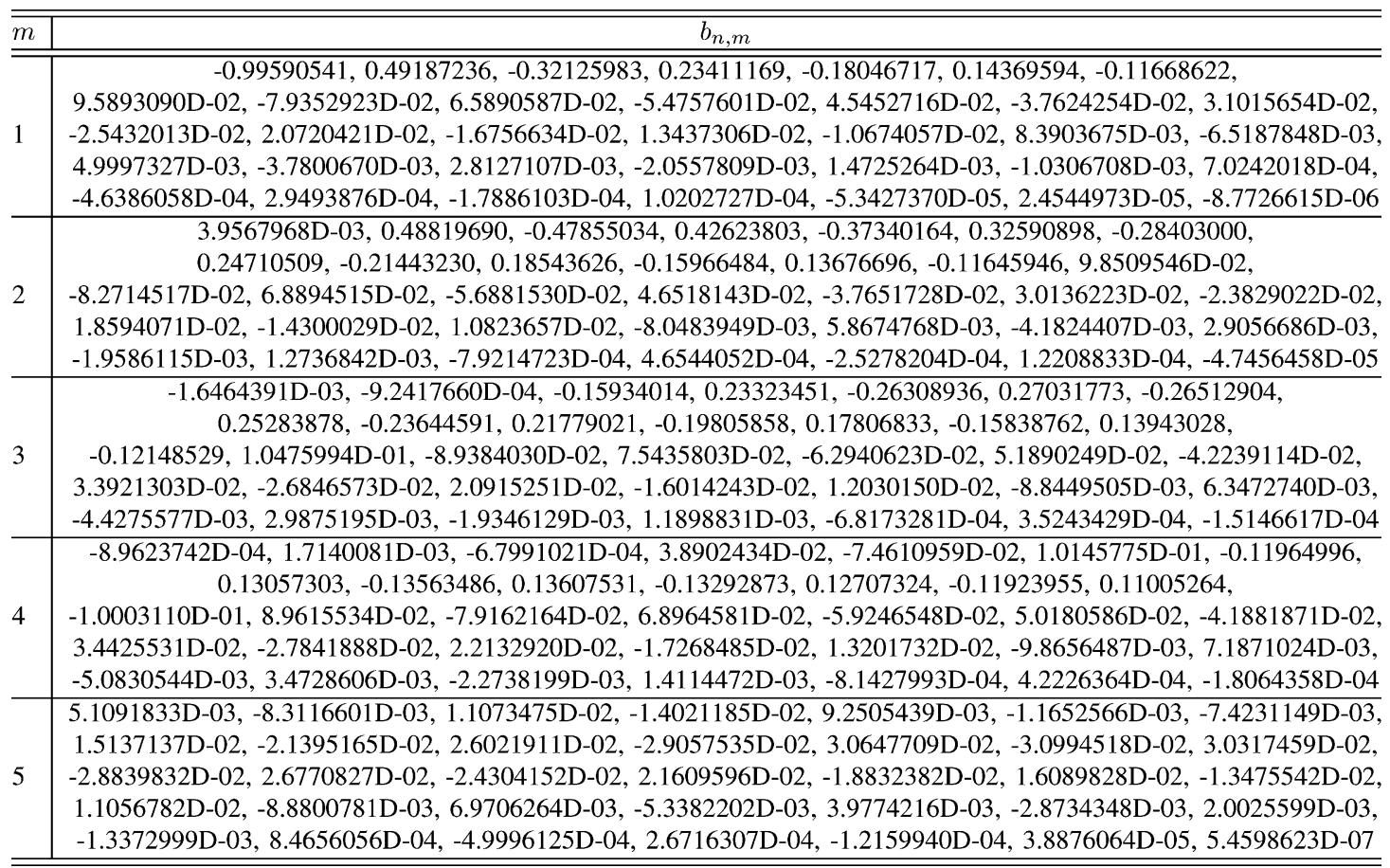

Fractional Delay Error

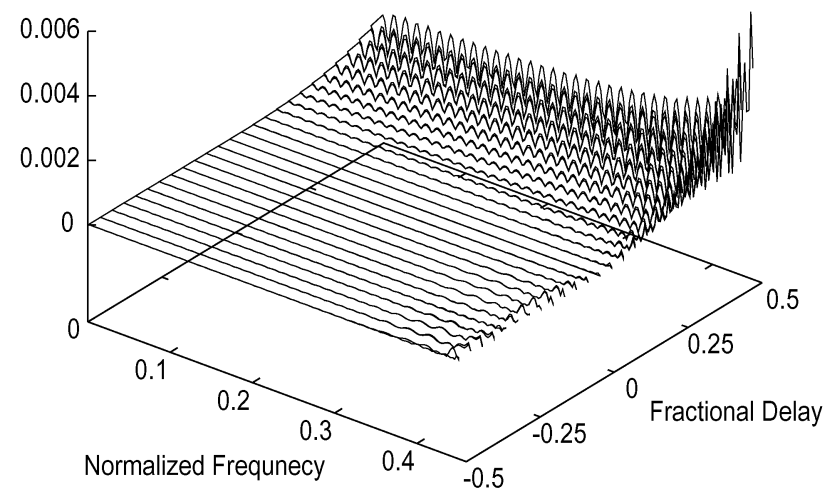

(a)

Phase Response Error

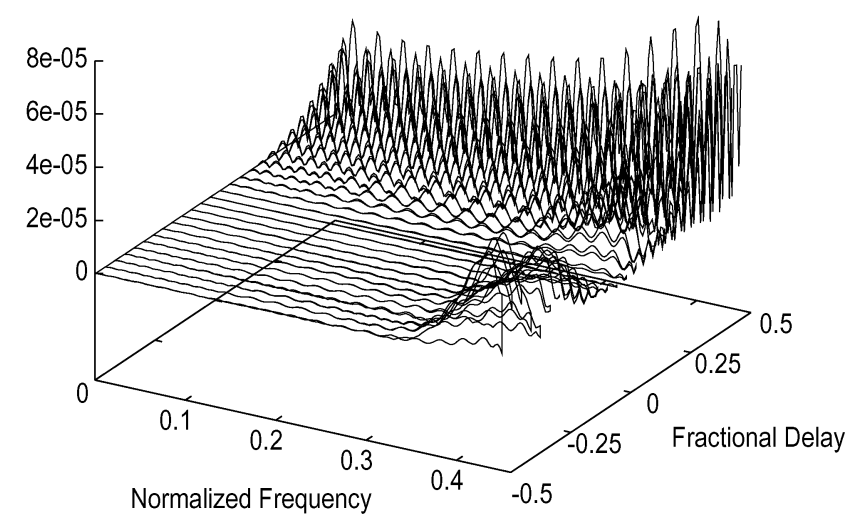

(b)

Fig. 2. CLS design with $\delta=0.0022$ : (a) the fractional-delay error, and (b) the phase-response error.

seen that the proposed CLS design (without weighting function) can produce both smaller error energy and maximum peak error

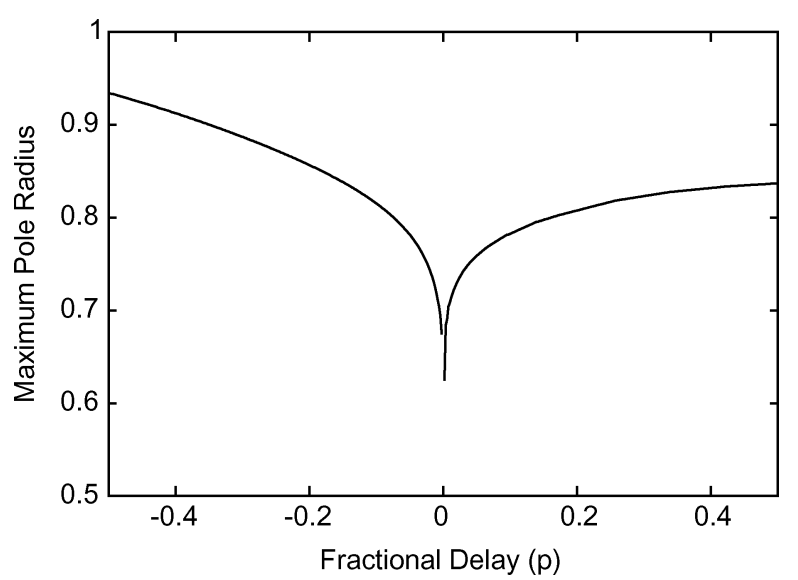

Fig. 3. Maximum pole radius (CLS design with $\delta=0.0022$ ).

of the fractional group delay than the non-iterative WLS design in [20] as well as the iterative WLS design in [19]. In particular, the maximum peak error of the fractional group delay is reduced to 0.005276 , which is $21 \%$ and $81 \%$ lower than the non-iterative WLS design in [20] and WLS design in [19], respectively.

In [21], a minimax method has been proposed for designing an all-pass VFD filter with minimum phase-delay. Generally speaking, it is not fair to compare an LS (or WLS) design with a minimax design because the objective of the minimax design is to minimize the worst-case (maximum) error, while the objective of the LS (or WLS) design is to minimize the total error energy. In some applications, the LS (or WLS) design is more important than the minimax design because the total error energy from the LS (or WLS) design is much smaller than that from the minimax design. On the other hand, if one wants to determine the worst-case error, the minimax design is preferred. Moreover, the delay used in the cost function in this paper is the group delay, $(d / d w) \theta(w, p)$, while the delay used in the cost 


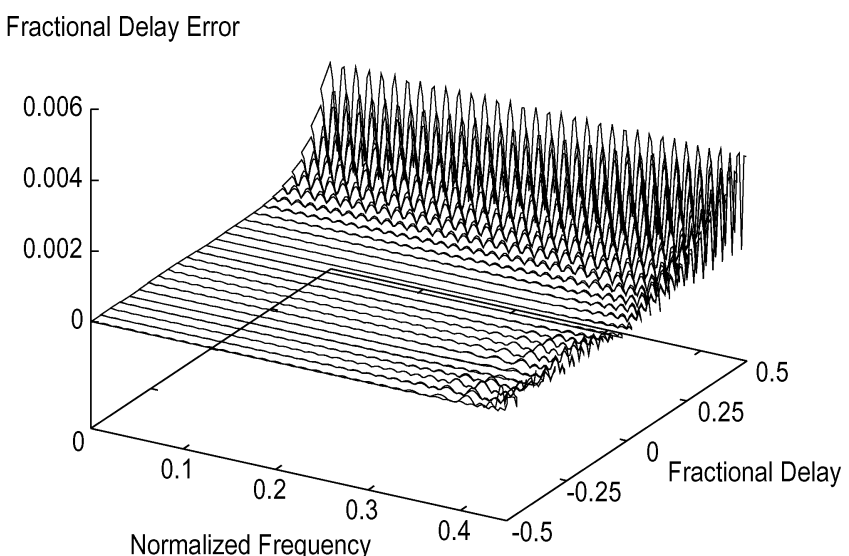

(a)

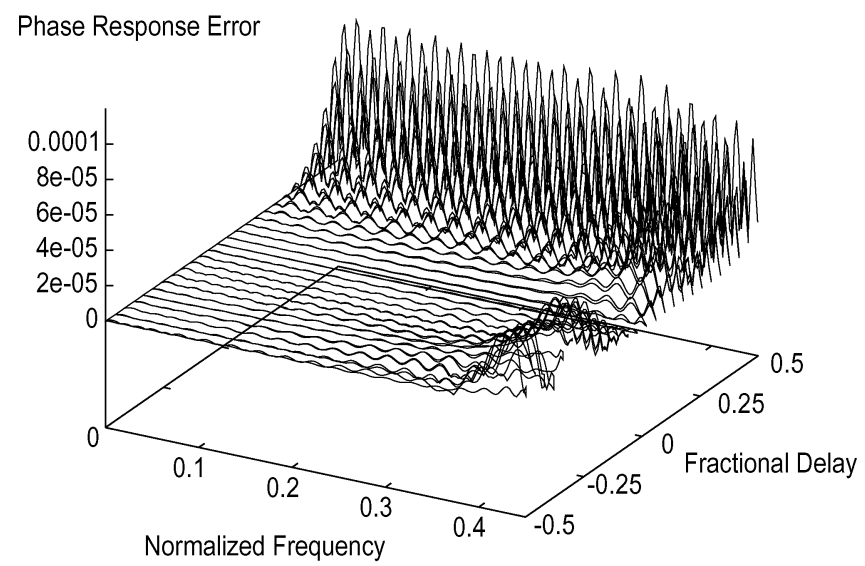

(b)

Fig. 4. WLS design with Problem WLS solved eight times. (a) Fractionaldelay error. (b) Phase-response error.

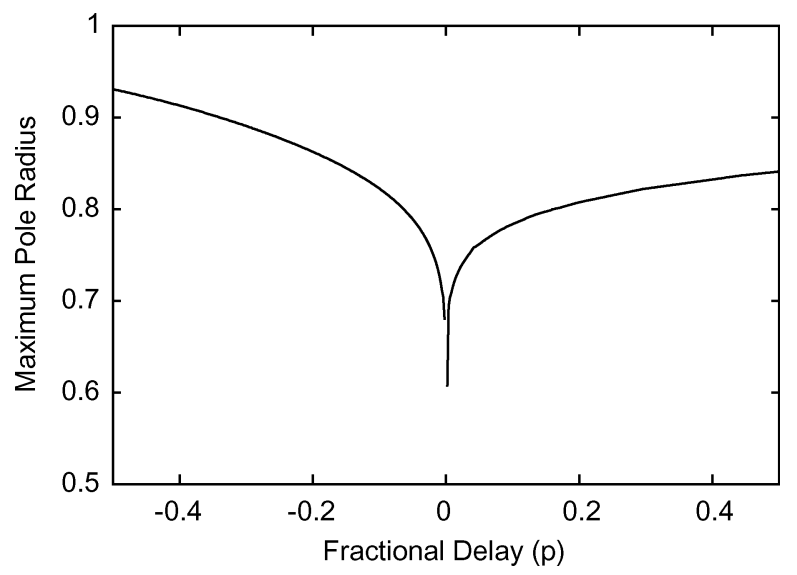

Fig. 5. Maximum pole radius (WLS design with Problem WLS solved eight times).

function in [21] is the phase delay, $\theta(w, p) / w$. Phase delay and group delay can be very different for some frequencies in a same design, since the filter, $H(w, p)$, designed to approximate the desired linear phase VFD filter, $H_{d}(w, p)$, is an all-pass filter which is usually highly nonlinear.

Fig. 2 depicts the absolute errors of the fractional group delay and the phase response from the proposed CLS design with $\delta=$ 0.0022 for the constraint (5). Table II lists the coefficients of the filter designed. Fig. 3 depicts the maximum radius of the poles of the designed all-pass VFD filter as the function of the fractional delay $p .500$ points are used for plotting the figure. Because the pole is always inside the unit circle, the designed all-pass VFD filter is stable as expected.

Fig. 4 depicts the absolute errors of the fractional group delay and phase response from the proposed WLS design with Problem WLS being solved 8 times. It can be seen that the maximum peak error of the fractional group delay is further reduced, and peak error is distributed more evenly than it is for the solution of the CLS design. It can also be seen that the proposed CLS design has a lower error energy of the fractional group delay than the proposed WLS design. If one seeks to minimize the error energy of the fractional group delay, the proposed CLS design is preferred. Fig. 5 depicts the maximum radius of the poles of the designed all-pass VFD filter using the proposed WLS design. The designed all-pass VFD filter is also stable as expected since the pole is always inside the unit circle.

\section{CONCLUSION}

We have presented a CLS design and a WLS design for allpass VFD filters, respectively. The methods have several important features. Firstly, the methods directly minimize the error energy of the fractional group delay. Consequently, the methods can produce smaller peak error and error energy of the fractional group delay than the methods published in the previous papers. Secondly, the highly nonlinear optimization problem formulated in the CLS design is converted to a linear LS optimization problem, which simply requires the solution of a linear system. Thirdly, an adaptive weighting function is introduced which is easy to calculate and leads to a further reduction in the peak error of the fractional group delay. Finally, it is reasonable to expect that the methods can be extended to the two dimensional all-pass VFD filter design.

\section{REFERENCES}

[1] C. W. Farrow, "A continuously variable digital delay element," in Proc. 1988 IEEE Int. Symp. Circuits Syst., Espoo, Finland, Jun. 1988, pp. 2641-2645.

[2] L. Erup, F. M. Gardner, and F. A. Harris, "Interpolation in digital modems-part II: Implementation and performance," IEEE Trans. Commun., vol. 41, no. 6, pp. 998-1008, Jun. 1993.

[3] S.-C. Pei and C.-C. Tseng, "A comb filter design using fractional-sample delay," IEEE Trans. Circuits Syst. II, Analog Digit. Signal Process., vol. 45, no. 6, pp. 649-653, Jun. 1998.

[4] K. Rajamani, Y.-S. Lai, and C. W. Farrow, "An efficient algorithm for sample rate conversion from CD to DAT," IEEE Signal Process. Lett., vol. 7, no. 10 , pp. $288-290$, Oct. 2000.

[5] G.-S. Liu and C.-W. Wei, "A new variable fractional sample delay filter with nonlinear interpolation," IEEE Trans. Circuits Syst. II, Analog Digit. Signal Process., vol. 39, no. 2, pp. 123-126, Feb. 1992.

[6] A. Tarczynski, G. D. Cain, E. Hermanowicz, and M. Rojewski, "WLS design of variable frequency response FIR filters," in Proc. 1997 IEEE Int. Symp. Circuits Syst., Hong Kong, Jun. 1997, pp. 2244-2247.

[7] W.-S. Lu and T.-B. Deng, "An improved weighted least-squares design for variable fractional-delay FIR filters," IEEE Trans. Circuits Syst. II, Analog Digit. Signal Process., vol. 46, no. 8, pp. 1035-1040, Aug. 1999.

[8] T.-B. Deng and W.-S. Lu, "Weighted least-squares method for designing variable fractional-delay 2-D FIR digital filters," IEEE Trans. Circuits Syst. II, Analog Digit. Signal Process., vol. 47, no. 2, pp. 114-124, Feb. 2000

[9] T.-B. Deng, "Discretization-free design of variable fractional-delay FIR filters," IEEE Trans. Circuits Syst. II, Analog Digit. Signal Process., vol. 48, no. 6, pp. 637-644, Jun. 2001 
[10] T. I. Laakso, V. Välimäki, M. Karjalainen, and U. K. Laine, "Splitting the unit delay: Tools for fractional-delay filter design," IEEE Signal Process. Mag., vol. 13, no. 1, pp. 30-60, Jan. 1996.

[11] T.-B. Deng, "Closed-form design and efficient implementation of variable digital filters with simultaneously tunable magnitude and fractional-delay," IEEE Trans. Signal Process., vol. 52, no. 6, pp. 1668-1681, Jun. 2004.

[12] T.-B. Deng, "SVD-based design and new structures for variable fractional-delay digital filters," IEEE Trans. Signal Process., vol. 52, no. 9, pp. 2513-2527, Sep. 2004.

[13] H. Johansson and P. Löwenborg, "On the design of ajustable fractionaldelay FIR filters," IEEE Trans. Circuits Syst. II, Analog Digit. Signal Process., vol. 50, no. 4, pp. 164-169, Apr. 2003.

[14] T.-B. Deng, "Design of arbitrary-phase variable digital filters using SVD-based vector-array decomposition," IEEE Trans. Circuits Syst. I, Reg. Papers, vol. 52, no. 1, pp. 148-167, Jan. 2005.

[15] T.-B. Deng, "Weighted-least-squares design of variable fractional-delay FIR filters using coefficient symmetry," IEEE Trans. Signal Process., vol. 54, no. 8, pp. 3023-3038, Aug. 2006.

[16] V. Välimäki and T. I. Laakso, "Principle of fractional-delay filters," in Proc. 2000 IEEE Int. Conf. Acoust., Speech, Signal Process., May 2000, pp. 3870-3873.

[17] M. Makundi, T. I. Laakso, and V. Välimäki, "Efficient tunable IIR and allpass structures," Electron. Lett., vol. 37, no. 6, pp. 344-345, Mar. 2001.

[18] M. Makundi, V. Välimäki, and T. I. Laakso, "Closed-form design of tunable fractional-delay allpass filter structures," in Proc. 2001 IEEE Int. Symp. Circuits Syst., Sydney, Australia, May 2001, pp. 434-437.

[19] C.-C. Tseng, "Design of 1-D and 2-D variable fractional-delay allpass filters using weighted least-squares method," IEEE Trans. Circuits Syst. I, Fundam. Theory Appl., vol. 49, no. 10, pp. 1413-1422, Oct. 2002.

[20] T.-B. Deng, "Noniterative WLS design of allpass variable fractionaldelay digital fiters," IEEE Trans. Circuits Syst. I, Reg. Papers, vol. 53, no. 2, pp. 358-371, Feb. 2006.

[21] J. Y. Kaakinen and T. Saramäki, "An algorithm for the optimization of adjustable fractional-delay all-pass filters," in Proc. 2004 IEEE Int. Symp. Circuits Syst., Vancouver, Canada, May 2004, pp. 153-156.

[22] M. Lang and T. I. Laakso, "Simple and robust method for the design of allpass filters using least-squares phase error criterion," IEEE Trans. Circuits Syst. II, Analog Digit. Signal Process., vol. 41, no. 1, pp. 40-48, Jan. 1994.

[23] T. Q. Nguyen, T. I. Laakso, and R. D. Koilpillai, "Eigenfilter approach for the design of allpass filters approximating a given phase response," IEEE Trans. Siganl Process., vol. 42, no. 9, pp. 2257-2263, Sep. 1994.

[24] X. Zhang and H. Iwakura, "Novel method for designing digital allpass filters based on eigenvalue problem," Electron. Lett., vol. 29, no. 14, pp. 1279-1281, Jul. 1993.

[25] Z. Jing, "A new method for digital all-pass filter design," IEEE Trans. Accoust., Speech, Signal Process., vol. 35, no. 11, pp. 1557-1564, Nov. 1987.

[26] K. E. Steiglitz and L. E. McBridge, "A technique for the identification of linear systems," IEEE Trans. Autom. Contr., vol. AC-10, no. 10, pp. 461-464, Oct. 1965.

[27] W.-S. Lu, S.-C. Pei, and C.-C. Tseng, "A weighted least-squares method for the design of stable 1-D and 2-D IIR digital filters," IEEE Trans. Siganl Process., vol. 46, no. 1, pp. 1-10, Jan. 1998.

[28] P. A. Regalia, "Comments on "A weighted least-squares method for the design of stable 1-D and 2-D IIR digital filters'," IEEE Trans. Siganl Process., vol. 47, no. 7, pp. 2063-2065, Jul. 1999.

[29] Y. C. Lim, J. H. Lee, C. K. Chen, and R. H. Yang, "A weighted leastsquares algorithm for quasi-equiripple FIR and IIR digital filter design," IEEE Trans. Signal Process., vol. 40, no. 3, pp. 551-558, Mar. 1992.

[30] A. Tarczynski, G. D. Cain, E. Hermanowicz, and M. Rojewski, "A wise method for designing IIR filters," IEEE Trans. Signal Process., vol. 49, no. 7, pp. 1421-1432, Jul. 2001.

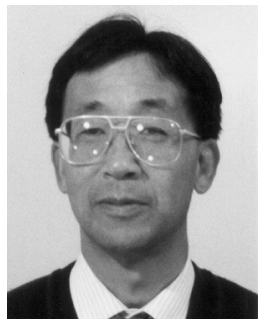

Wei Rong Lee received the Ph.D. degree in applied mathematics from Curtin University of Technology, Perth, WA, Australia, in 1999.

From 1999 to 2001, he worked as a Research Associate in the Department of Mathematics and Statistics, Curtin University of Technology. In 2001, he joined Western Australian Centre of Excellence in Industrial Optimisation (WACEIO), Curtin University of Technology, as a Research Fellow. His main fields of research are theory and applications of optimization and optimal control, in particular, the applications in signal processing, numerical analysis, stochastic processes with applications to hidden Markov model.

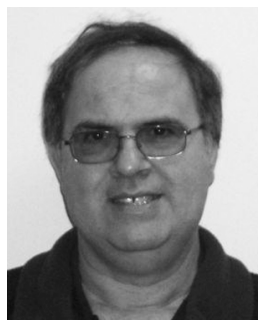

Lou Caccetta received the B.Sc. and Ph.D. degrees from the University of Western Australia, Perth, WA, Australia.

Following this, he spent four years in Canada, mostly in the Department of Combinatorics and Optimisation at the University of Waterloo, Waterloo, ON, Canada. He joined Curtin University of Technology, Perth, WA, Australia, as Lecturer in Operations Research in 1981 and was promoted to Senior Lecturer and Associate Professor in 1986 and 1990, respectively. He obtained a Personal Chair in 1992 with the title, Professor of Mathematics. He served for ten years as Head of the School of Mathematics and Statistics from June 1994 to July 2004. He established the Western Australian Centre of Excellence in Industrial Optimisation (WACEIO) at Curtin University of Technology in 2001. WACEIO undertakes industry focused $R \& D$ and is a recognized provider of cutting edge industrial optimization technology. His research interests include: computational algorithms, combinatorial mathematics (graph theory and network optimization), combinatorial optimization (integer programming), industrial modelling and operations research. His research projects span theory, methods and applications. He has developed, implemented and tested a number of optimization routines for solving large industrial problems. He is on the Editorial Board of nine journals and is a Reviewer and Referee for a number of journals and funding agencies. He has supervised to completion $22 \mathrm{Ph} . \mathrm{D}$ 's, five Master's, and is currently supervising $12 \mathrm{Ph} . \mathrm{D}$. students. He has organized a number of conferences/workshops. He has published over 200 refereed papers and has won a number of competitive research grants. He has extensive experience in contract $R \& D$ and industrial consultancy.

Dr. Cacetta is a member of a number of professional affiliations and has severed as the National President of the Australian Society for Operations Research. He won the Deans Medallion for Distinguished Research, Faculty of Science, Curtin University of Technology in 2000 and is a recipient of the Vice-Chancellors Award for Excellence.

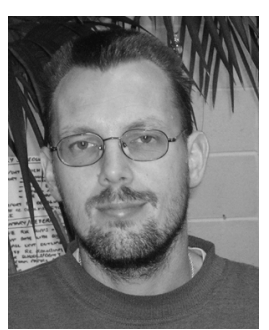

Volker Rehbock received the Ph.D. degree in applied mathematics from the University of Western Australia, Perth, WA, Australia, in 1994.

Since 1995, he has been with the Department of Mathematics and Statistics, Curtin University of Technology, Perth, WA, Australia. His main interests are in the modelling and numerical solution of dynamic optimization problems, particularly those involving variable switching times. He has co-authored over 60 refereed publications in the areas of optimization and optimal control. 